\title{
Microstructure and Rheology of Lamellar Liquid Crystalline Phases
}

\author{
P. Versluis* and J . C. van de Pas \\ Unilever Research Laboratorium Vlaardingen, Olivier van Noortlaan 120, \\ 3133 AT Vlaardingen, The Netherlands \\ J. Mellema \\ Rheol ogy Group, Faculty of Applied Physics, University of Twente, P.O. Box 217, \\ 7500 AE Enschede, The Netherlands
}

Received March 7, 1997. In Final Form: August 1, 1997

\begin{abstract}
We have investigated the microstructure and rheological properties of ternary surfactant mixtures in a salt solution. Thesurfactants were $6 \%$ sodium 4-dodecyl benzenesulfonate, $3 \% \mathrm{C}_{13-15}$ ethoxylated alcohol with seven ethylene oxide (EO) units and $1 \% \mathrm{C}_{13-15}$ ethoxylated alcohol with $2,4,7,9,11,14,20$, or 25 EO units. The salt solution was $10 \%$ nitrilotriacetate $\cdot \mathrm{H}_{2} \mathrm{O}$. Microstructural investigations (electron microscopy, light microscopy, confocal laser microscopy, conductivity measurements, and centrifugation) show that at rest the samples containing thesurfactant with $2 \mathrm{EO}$ to $9 \mathrm{EO}$ units are dispersions of Iamellar droplets (curved surfactant bilayers). The samples containing the surfactant with 11 EO to 25 EO units show a continuous lamellar structure (sheets of surfactant bilayers) with a small amount of lamellar droplets present. The change in several rheological parameters reflects this change in microstructure. The power law index from flow experiments at low shear rates changes from 0.1 for thelamellar dispersions to 0.4 for the continuous lamellar phases. Similar changes are observed in shear modulus and in the limiting strain for linear viscoelastic behavior. The continuous lamellar phase is converted to droplets by shearing at rates above $1 \mathrm{~s}^{-1}$. The continuous lamellar structures will recover in about a week when the samples are all owed to relax. The nature of the dropl ets is highly dynamic. Confocal laser microscopy shows small fluctuations in droplet shape on a time scale of about $100 \mathrm{~s}$. This time coincides with a characteristic time of around 100 s pertaining to a (shallow) peak in G".
\end{abstract}

\section{Introduction}

Many (commercially available) surfactants used in detergent products have a tendency, when dissolved in water or a salt solution, toform liquid-crystallinelamellar phases. ${ }^{1}$ Theselamellar phases consist of stacked bilayers of alternating layers of ordered surfactant molecules and solvent. The surfactants in the bilayers are organized in such a way that the hydrophobic tails of the surfactant molecules are at the center of the lamellae and the hydrophilic parts of the molecules are in contact with the solvent layer. A schematic representation of a lamellar phase is given in Figure 1. The disadvantage of this type of representation is that it is static. It does not do justice to the dynamic nature of these phases, with undulating planes and surfactant molecules migration in and out of the bilayer.

Lamellar phases are known to exist in at least two configurations. In addition to a planar or continuous lamellar phase, where the bilayers are ordered in sheets, ${ }^{2}$ lamellar phases exist with the bilayers ordered in closed concentric shells. ${ }^{3-5}$ The structural units in the latter phase are often referred to as "onions", multilamellar vesicles, or lamellar droplets. From previous studies it appears that the onions are stable even at low

\footnotetext{
$\otimes$ Abstract published in AdvanceACS Abstracts, September 15, 1997.

(1) van de Pas, J. C. A study of the physical properties of lamellar liquid-crystallinedispersions. Ph.D. Thesis, Rijksuniversiteit Groningen, 1993; Chapters 1 and 2.

(2) Bleasdale, T. A.; Tiddy, G. J T. In The Structure, Dynamics an Equilibrium Properties of Coll loidal Systems; Bloor, D. M., Wyn-J ones, E., Eds.; Kluwer Academic Publishers: TheNetherlands, 1990; pp 397414.

(3) J urgens, A. Tenside Surfactants Deterg. 1989, 26, 222.

(4) van de Pas, J. C. Tenside Surfactants Deterg. 1991, 28, 158.

(5) Hoffmann, H.; Thunig, C.; Schmiedel, P.; Munkert, U. Langmuir 1994, 10, 3972.
}

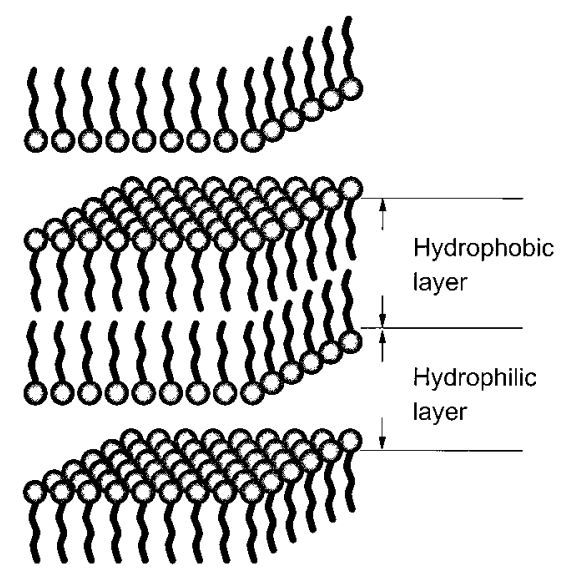

Figure 1. Schematic representation of surfactant molecules arranged in bilayers.

surfactant concentrations when prepared from mixtures of nonionic and anionic surfactants at sufficiently high salt concentrations. ${ }^{6}$

When the surfactant head groups are well hydrated and the alkyl layer is fluid, these phases can swell considerably, indicating repulsion between thelayers. The spacing between the bilayers can be orders of magnitude larger than the typical dimensions of the hydrophilic surfactant head group. ${ }^{7}$ This is thought to be caused by thermal undulation ${ }^{8}$ of thelayers. This swelling behavior can lead to a space-filling arrangement even at low to moderate surfactant concentrations. When, for onions,

(6) Sein, A.; Engberts, J . B. F. N.; van Linden, E.; van de Pas, J . C Langmuir 1993, 9, 1714.

(7) Strey, R.; Schomäcker, R.; Roux, D.; Nallet, F.; Olsson, U.J .Chem. Soc. Faraday Trans. 1990, 86, 2253.

(8) Helfrich, W. Z. Naturforsch. 1978, 33A, 305. 
the volume fraction of lamellar phase $\left(\phi_{\mathrm{lam}}\right)$ is at or just above the random maximum packing of monodisperse spheres (0.63), it provides an excellent matrix for suspending solid particles, enabling the formulation of, e.g., fabric washing liquids. ${ }^{3,4}$

Onions and continuous lamellar phases haveinteresting rheological properties. It has, for instance, been reported that a continuous lamellar phase, prepared from AOT in brine solutions or from SDS, pentanol, water, and dodecane, can be converted to onions by shear ${ }^{9-11}$ which will relax back to continuous lamel lar in a few days to several months, depending on the composition. Strong shear hysteresis in systems containing 5\% AOT in water have been reported. ${ }^{12}$ Thehysteresis is thought to beassociated with a shear-induced phase transition.

We have studied the microstructure and rheological properties of a typical liquid detergent composition. A small change in the average ethylene oxide chain length of the nonionic surfactant caused transition of onions to a mainly continuous lamellar phase. The liquids exhi bit striking non-Newtonian rheol ogical behavior. They show elasticity at small deformations, possibly due to the deformability of the droplets and theinteractions between thedroplets. Furthermore, theliquids show marked shear thinning.

Theresearch described in this paper was carried out in an industrial research laboratory, using industrial raw materials. Altough theuse of commercial gradematerials may lead to a relatively large scatter in experimental results, the translation to industrial products and processes is as simple and direct as possible.

\section{Experimental Section}

2.1. Materials. The anionic surfactant used was Marlon AS-3, from Huls, a commercial preparation of DoBS-acid, which is a mixture of $98.0-98.5 \%$ isomerically impure 4-(sec-dodecyl)benzenesul fonicacids, about 1.5\% non-sul fonated organic matter and $0.3 \%$ sulfuric acid. The nonionic surfactants were sel ected from the range of Synperonics from $\mathrm{ICl}$ which are $\mathrm{C}_{13-15}$ ethoxylated alcohols, with an average of $2,4,7,9,11,14$, and $20 \mathrm{~mol}$ of ethylene oxides per mol. We also used a $\mathrm{C}_{13-15}$ ethoxylated alcohol with 25 ethylene oxide monomer units on average (Ukanil 102, from Ugine Kuhlmann). It should be stressed that the EO lengths given represent an average value and that the distribution in lengths is very broad.

The salt used was nitrilotriacetate $\cdot \mathrm{H}_{2} \mathrm{O}$, NTA (Trilon A92, from BASF).

All materials were used without further purification.

Model samples were prepared by dissolving the surfactants in demineralized water under stirring at $50^{\circ} \mathrm{C}$, until a clear isotropic surfactant solution was obtained. A sufficient amount of sodium hydroxidewas added to neutralizetheacid surfactant. After addition of the NTA, theliquid was allowed to cool to room temperature under stirring. Finally the $\mathrm{pH}$ of the liquid was adjusted to around 8 by adding $\mathrm{NaOH}$ solution. The samples were stored for at least 1 month in closed plastic bottles at room temperature before any measurement was made.

The surfactant concentration in the samples was $10 \%$ by weight, with an anionic:nonionic weight ratio of $6: 4$ (based on the sodium salt). The base formulation contains $6 \% \mathrm{Na}-\mathrm{DoBS}$, $3 \%$ Synperonic A7 (7 mol of ethylene oxide per mol of surfactant, abbreviated as 7EO), and $1 \%$ nonionic surfactant with $\times E O$, where $x=2,4,7,9,11,14,20$, or 25 . The sample codes used in this paper indicate the variation in the $\mathrm{EO}$ chain length of the latter nonionic surfactant. The NTA level is $10 \%$ by weight.

(9) Diat, O.; Roux, D. J . Phys. II 1993, 3, 9.

(10) Diat, O.; Roux, D.; Nallet, F.J . Phys. II, 1993, 3, 1427.

(11) van der Linden, E.; Hogervorst, W. T.; Lekkerkerker, H. N. W. Langmuir 1996, 12, 3127.

(12) Valdés, M.; Manero, O.; Soltero, J . F. A.; Puig, J . E. J . Colloid Interface Sci. 1993, 160, 59.
2.2. Measurements. All measurements were performed at $25 \pm 0.1{ }^{\circ} \mathrm{C}$ except the light microscopic and confocal laser microscopic observations, which were done at room temperature $\left(20-25^{\circ} \mathrm{C}\right)$.

Electron micrographs of all samples were obtained by freeze fracture/etching/heavy metal replication. The $5 \mu \mathrm{L}$ aliquots of the samples used were slam-frozen in a Reichert MM80 slam freezer and transferred to a Cressington CFE 50 freeze fracture and etching apparatus. After fracturing, thesamples wereetched for $2 \mathrm{~min}$ at $-100^{\circ} \mathrm{C}$ and replicated with a $2 \mathrm{~nm}$ layer thickness of $\mathrm{W} / \mathrm{Ta}$ and a $7 \mathrm{~nm}$ carbon backing. The replicas were washed and observed in a Philips CM 12 transmission el ectron mi croscope (TEM) at $120 \mathrm{kV}$ accelerating voltage. Digital imageacquisition was done with a Gatan slow scan CCD camera, type 694, and printing by a Fujix Pictographic 3000 printer. Best results were obtained when, previous to freezing, the sampleholder with the sample in place was allowed to rest for several minutes in a saturated water vapor atmosphere to prevent drying of the sample. The micrographs were inspected visually to assess the microstructure of the samples.

Samples were also inspected with a Zeiss Axioplan light microscope using polarized light. A small drop of material was placed on a microscope slide and quickly covered with a cover glass. The sampleflows to fill the gap between theslideand the cover glass. The samples were observed for up to a few hours after cessation of flow, until no change in microstructure could be detected anymore. Images of relevant structures were obtained using a Sony CCD video camera and a video printer. To examine the sample in an unperturbed surrounding, we also used confocal light mi croscopy wherethesamplecan beobserved, several micrometers away from the surface of the hol ding vessel into the bulk of the sample. For the confocal laser microscopy, a small vol ume of a selected samplewas treated with a few drops of a $0.1 \%$ aqueous solution of rhodamine. This fluorescent agent preferentially adsorbs in thehydrophobic domains of thebilayers. ${ }^{6}$ The sample was allowed to rest for a few days in the sample holder to allow relaxation of theaccumulated stresses dueto the handling of thesamples. Subsequently a timeseries of 40 images was recorded with 20 and 100 s intervals. The sampl es used in light microscopy and confocal laser microscopy were sealed to avoid changes in composition due to evaporation.

Tocal culatethevol umefraction lamel lar droplets, theel ectrical conductivity of thesamples was measured in a cell with platinized electrodes using a Radiometer CDM 83 conductivity meter.

The samples werecentrifuged for $16 \mathrm{~h}$ at $40000 \mathrm{~g}$. The height of separated layers, if any, was measured with a ruler. This allows a rough calculation of the volume of the layers.

Dynamicrheol ogical measurements were madewith a Bohlin VOR constant shear rate rheometer equipped with a smooth couette geometry ( $C 25$, bob diameter $25 \mathrm{~mm}$, gap $0.125 \mathrm{~mm}$ ) in the linear viscoelastic region. The sample was carefully introduced into the cylindrical geometry, avoiding high rates of deformation as much as possible. Subsequently several frequency sweeps from $10-0.001-10 \mathrm{~Hz}$ were done on the sample. After a maximum of four sweeps, no further systematic changes in $\mathrm{G}^{\prime}$ and $\mathrm{G}^{\prime \prime}$ occurred, indicating that the sample had fully relaxed. Data from the fifth and sixth sweep were used. The strain was limited to 0.02 , which is in the linear region.

Measurements of viscosity as function of shear rateweremade on a Bohlin CS rheometer, using a constant shear rate software package. Therheometer was equipped with a serrated concentric cylinder setup (bob diameter $25 \mathrm{~mm}$, gap $0.125 \mathrm{~mm}$, grooves of $0.1 \mathrm{~mm}$ ) to prevent wall slip. ${ }^{3}$ The same precautions weretaken as for the dynamic measurements, and the sample was allowed torelax beforeany measurements weremade. Shear rates ranged from 0.065 to $328 \mathrm{~s}^{-1}$ in 40 logarithmically spaced steps. The measuring time was $80 \mathrm{~s}$ at the lowest shear rate decreasing in proportional steps to $20 \mathrm{~s}$ at the highest shear rate. Two updown curves were recorded (up and down refer to the direction of change of the shear rates).

For all samples, dynamic measurements were also performed after a certain shear rate had been imposed on the sample for $300 \mathrm{~s}$. The shear rates used were $0,5.8 \times 10^{-3}, 5.8 \times 10^{-2}, 5.8$ $\times 10^{-1}, 5.8,58$, and $367 \mathrm{~s}^{-1}$, respectively. The measurements were performed on the Bohlin VOR. The dynamic moduli of two samples were measured at $1 \mathrm{~Hz}$ after each sample was sheared for 300 s at $328 \mathrm{~s}^{-1}$ for an extended period of time (about 1 week). 


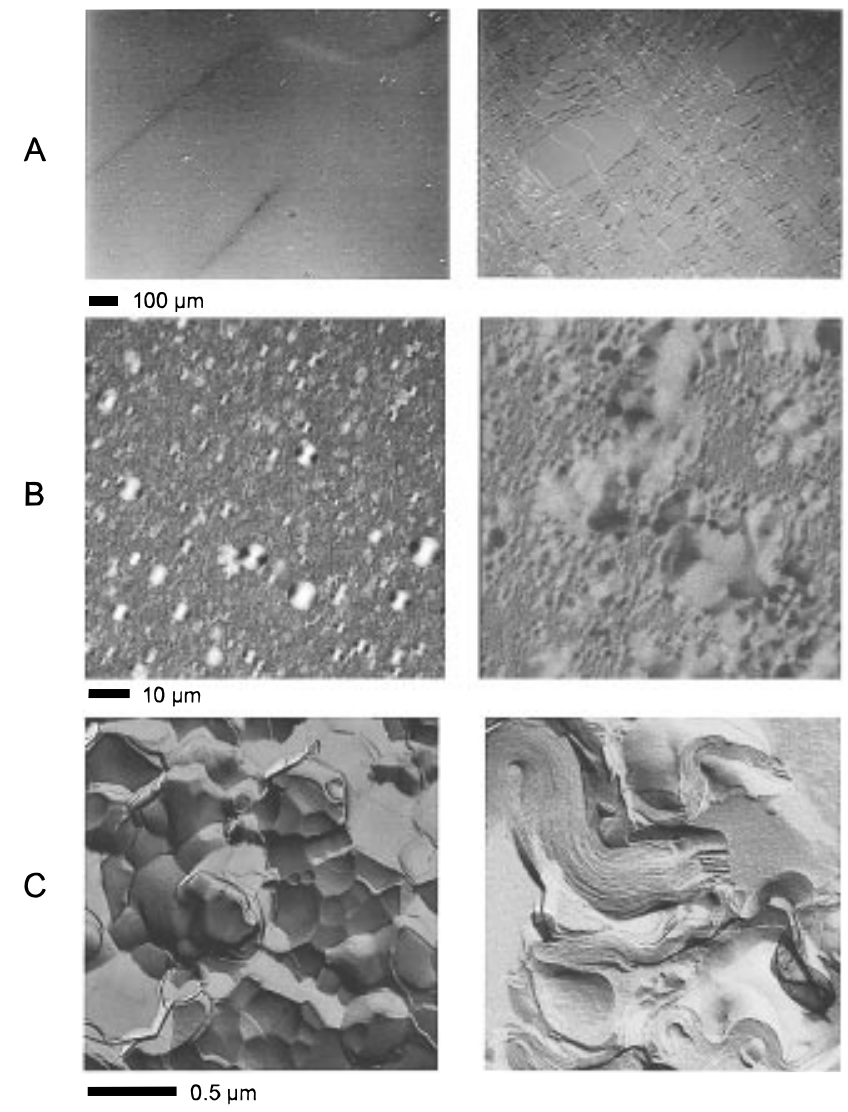

Figure 2. From top to bottom, polarized light (A), confocal laser (B), and electron microscopic (C) pictures of relevant structures observed in 4EO (left column) and 14EO (right col umn) samples.

The samples were inspected microscopically after shearing at $367 \mathrm{~s}^{-1}$ for $300 \mathrm{~s}$ to examine the effect of shear on the microstructure.

\section{Results and Discussion}

For clarity only a limited number of photographs and curves are presented. The observations made are well represented by thesamples 4E O and 14E O (abbreviations explained in section 2.1). Wewill, however, present results for all samples where appropriate.

3.1. Microstructure. Typical examples of microstructures are given in Figure 2. On the left-hand side, representative images for thesample with 4E $\mathrm{O}$ are given, and on the right-hand side, images for the sample with 14E O can befound. Thetop, middle, and bottom pictures were obtained with light microscopy, confocal laser microscopy, and electron microscopy, respectively.

Polarized light microscopy shows that all samples contain some dropl et structures with Maltese extinction crosses. This is a clear indication of the presence of multilamellar droplets. The droplet sizes are above 0.5 $\mu \mathrm{m}$ becausethey wereobserved in opti cal mi croscopy with a detection limit of around $0.5 \mu \mathrm{m}$ because of the wavelength of thelight used. In thesedroplets, alternating surfactant-water bilayers are present as concentric spherical shells. Also some birefringence was detected, especially when the sample flows around an entrapped air bubble. This birefringence is associated with the existence of small lamellar droplets, just below the resolution limit of the microscope. ${ }^{1}$ The dark and light streaks present in the top left image in Figure 2 indicate the presence of small birefringent structures, just below the detection limit of the microscope.
Some samples show a drastic change in microstructure when they are left to rest for several minutes. The appearancechanges from birefringent with somelamellar droplets to platelike, with some lamellar droplets still present. This is the casefor thesamples $11 \mathrm{E} O$ and higher. F or the samples 2EO to 9EO no change in appearance with time is observed. The light microscopic image for the sample with 14E O (top right in Figure 2) shows, next to some droplets, large areas of flat, platelike structures, extending over $100 \mu \mathrm{m}$ or more. The edges of the plates are birefingent. They are either light or dark, depending on the orientation of the sample. When the orientation of the sample under the light microscope is changed, the light edges become dark and vice versa. This means that the observed plates must consist of multiplebilayers since the thickness of a single bilayer is too small (on the order of $10 \mathrm{~nm}$ ) to be visible by light microscopy.

The microstructural information obtained with the confocal laser microscope confirms to a large extent the view developed on the basis of the ordinary light microscopy. The pictures show a filled array of mainly spherical droplets for samples $2 \mathrm{EO}$ to $9 \mathrm{EO}$ and more extended structures for $11 \mathrm{E} \mathrm{O}$ to $25 \mathrm{EO}$. We interpret the apparent difference between these largestructures under the light microscope and in confocal laser microscopy to be caused by the sample preparation method. In light microscopy, large structures are flattened between the glass surfaces of the slide and cover glass. Therefore, these structures will no longer show internal structure. Also some very largedroplets with a typical size of a few micrometers are present. These droplets show Maltese crosses as seen in ordinary light microscopy, because of the radial symmetry present.

The electron micrographs for the samples with $2 \mathrm{EO}$ until 9EO confirm that these samples contain many, basically spherical, highly packed droplets. In the case of $4 \mathrm{EO}$ the high degree of packing leads to deformation of the droplets into a "honeycomb" like arrangement. Electron micrographs of this type of packing for nearly monodisperse lamellar dropl ets are shown elswhere. ${ }^{13}$ In the samples 2EO, 7EO, and 9E O the deformation of the droplets is much less noted. We have no explanation for this difference in appearance. The electron micrograph of the sample with 14EO shows continuous lamellar platelike structures. These structures are not perfectly flat but show a curved substructure like in the confocal laser microscopy images. In several places on the photograph theindividual surfactant bilayers arevisible. It is interesting to see that the continuous lamellar layer terminates at the edge by closure of not more than a few bilayers, showing locally a high curvature.

Close examination of all the micrographs reveals that the systems with surfactant EO chain length of 9 and smaller aredispersions of lamellar droplets. F or EO chain lengths equal to 11 and larger, thestructurechanges quite abruptly to primarily continuous lamellar with an occasional droplet.

We al so used the confocal laser microscope to record a series of images taken with a constant timeinterval. When such a sequence, which extended over a period of more than one hour, is played back in a few seconds, one can clearly see the dynamic nature of the samples containing only droplets. The individual droplets show continuous shape fluctuations. The eye is sensitive enough to detect thesechanges in shape when thepictures are put together in a movie, but it is impossible to see this on static photographs. We estimate the magnitude of the fluctua-

(13) Gulik-Krzywicki, T; Dedieu, J . C.; Roux, D; Degert, C; Laversanne, R. Langmuir 1996, 12, 4668. 
Table 1. Volume Fraction Lamellar Phase Calculated from Conductivity $\left(\phi_{\mathrm{lam}}^{\mathrm{b}}\right)$ and Centrifugation $\left(\phi_{\text {lam }}^{\mathrm{c}}\right)$, Respectively

\begin{tabular}{cll}
\hline sample $^{\mathrm{a}}$ & $\phi_{\text {lam }}^{\mathrm{b}}$ & $\phi_{\text {lam }}^{\mathrm{c}}$ \\
\hline 2EO & 0.84 & 1 \\
4EO & 0.73 & 1 \\
7EO & 0.54 & 1 \\
9EO & 0.49 & 1 \\
11EO & 0.39 & 0.91 \\
14EO & 0.30 & 0.86 \\
20EO & 0.35 & 0.83 \\
25EO & 0.35 & 0.82
\end{tabular}

a The nEO labels refer to the composition of the samples as explained in section 2.1.

tions to be, at maximum, about $5 \%$ of the size of a typical droplet. On one occasion, for sample 4EO, we observed that the shape fluctuations lead to the breakup of a large droplet into two smaller droplets.

From the electrical conductivity, the volume fraction lamellar phase was calculated using the Bruggeman ${ }^{14}$ equation adapted for lamellar dispersions ${ }^{4}$

$$
\left(\frac{\kappa-\kappa_{\mathrm{lam}}}{\kappa_{\mathrm{el}}-\kappa_{\mathrm{lam}}}\right)\left(\frac{\kappa_{\mathrm{el}}}{\kappa}\right)^{(1 / 3)}=1-\phi_{\mathrm{lam}}
$$

in which $\kappa$ denotes the el ectrical conductivity of the total system, $\phi_{\text {lam }}$ is the volumefraction lamellar phaseand the subscripts lam and el refer to the dispersed lamellar droplets and the continuous electrolyte solution respectively. For the conductivity of the dispersed lamellar droplets a value of $0.8 \mathrm{mS} / \mathrm{cm}$ was used 4 whereas for the continuous salt solution, the conductivity of a salt solution prepared without the surfactants was used.

The volume fraction lamellar phase can also be estimated from the volume of the clear layer that forms after centrifugation. We have assumed that, unlike the case of dispersions of solid particles, thedroplets in our systems are deformable enough to facilitate packing to a volume fraction of near one. In some cases, for samples 14EO to $25 \mathrm{E}$ O, a very thin isotropic top layer was detected. The layers had a slight yellow color, which is an indication of the presence of a small amount of surfactant in this layer. The original samples showed no such a layer, even after being allowed to stand for more than 6 months at room temperature. The presence of surfactant in the top layer is therefore most likely a result of the high g-force during centrifugation. The results of both methods are listed in Table 1.

Previously it was found that for onion phases the differencein volumefractions between centrifugation and conductivity is at most $10 \%{ }^{3}$ The assumption that the lamellar droplets can pack to a volume fraction of 1 may be too optimistic. The maximum attainable packing is probably lower. Despite this, there still exists a rather large discrepancy between thetwo results. The observed change in microstructure probably explains to a large extent the deviation between the volume fractions calculated from conductivity and from centrifugation. For the calculation of the volume fraction of the dispersed lamellar phasefrom conductivity, oneassumes this phase to be present as lamellar droplets with low conductivity. As soon as the continuous lamellar phase is present, this is nolonger thecase. When thecontinuous lamellar layers are positioned perpendicular with respect to the conductivity electrodes, the dispersed phase will have a conductivity that is much higher, probably on the order of the

(14) Bruggeman, D. A. G. Ann. Phys. 1935, 24, 636.

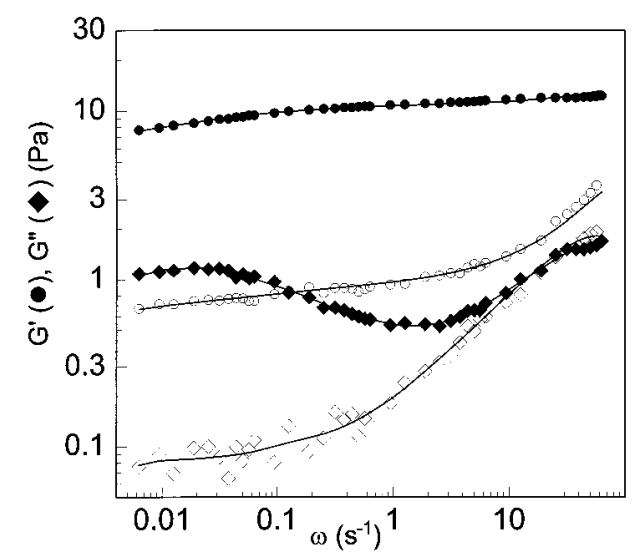

Figure 3. Dynamic moduli as function of frequency for sample 4EO (solid symbols) and 14EO (open symbols).

conductivity of the continuous salt solution. The same line of reasoning was followed elswhere. ${ }^{1}$

We ther efore conclude that the volume fraction calculated from conductivity is at best indicativefor thevolume of lamellar droplets, whereas the volume fraction from centrifugation is the sum of both continuous and droplet lamellar phases.

3.2. Rheology. 3.2.1. Linear Experiments. Two typical examples of dynamicmoduli ( $\mathrm{G}^{\prime}$ and $\mathrm{G}^{\prime \prime}$ ) as function of frequency are given in Figure 3. Again the 4E O data are representativein magnitudeand shape of thedata for samples $2 \mathrm{E} O$ until $9 \mathrm{EO}$ whereas 14EO is representative for $11 \mathrm{EO}$ until 25EO. Successiveruns with fresh samples and different torsion bars give a standard deviation for $\mathrm{G}^{\prime}$ of $8 \%$ and for $\mathrm{G}^{\prime \prime}$ of $15 \%$. The larger error in $\mathrm{G}^{\prime \prime}$ is inherent tothe measuring method when $\mathrm{G}^{\prime}$ is much larger than $\mathrm{G}^{\prime \prime}$, as is the case here.

For the samples 2EO to 9EO one can clearly detect the onset of a broad relaxation transition at low frequencies. The position of this transition shifts to higher frequencies with increasing EO length. From the slope of G" as function of frequency after thetransition, it can bededuced that this transition is not the result of a single relaxation mechanism, with a single characteristic time. In that case one would expect G" to decrease, in a double log plot with a slope of -1 . For the sample with $4 \mathrm{EO}$, the characteristictimeat which thetransition occurs is of the order of $100 \mathrm{~s}$ and coincides with the times associated with the fluctuations of the droplet shape as observed with confocal light microscopy. For samples 11EO until $25 \mathrm{EO}$, with a prevailing continuous lamellar phase, this transition is absent. For all samples, the onset of a relaxation process at high frequencies is observed.

The data can bereduced by fitting to a linear viscoel astic model with

$$
G^{\prime}=G_{0}+\sum_{p=1}^{i} \frac{G_{p} \omega^{2} \tau_{p}^{2}}{1+\left(\omega \tau_{p}\right)^{2}}
$$

and

$$
\mathrm{G}^{\prime \prime}=\sum_{\mathrm{p}=1}^{i} \frac{\mathrm{G}_{\mathrm{p}} \omega \tau_{\mathrm{p}}}{1+\left(\omega \tau_{\mathrm{p}}\right)^{2}}
$$

An apparent plateau in $\mathrm{G}^{\prime}$ for $\omega \rightarrow 0\left(\mathrm{G}_{0}\right)$ was included for curve-fitting purposes although eventually for very low frequencies one expects $G^{\prime}$ to drop again with decreasing frequency. 
Table 2. Results of the Fit of Dynamic Moduli to the Eight-Parameter Model

\begin{tabular}{lllllllll}
\hline sample & $\mathrm{G}_{1} / \mathrm{Pa}$ & $\mathrm{G}_{2} / \mathrm{Pa}$ & $\mathrm{G}_{3} / \mathrm{Pa}$ & $\mathrm{G}_{4} / \mathrm{Pa}$ & $\mathrm{G}_{5} / \mathrm{Pa}$ & $\mathrm{G}_{6} / \mathrm{Pa}$ & $\mathrm{G}_{7} / \mathrm{Pa}$ & $\mathrm{G}_{0} / \mathrm{Pa}$ \\
\hline 2EO & 3.23 & 0.23 & 0.36 & 0.19 & 0.49 & 0.55 & 1.65 & 8.58 \\
4EO & 2.95 & 0.59 & 0.32 & 0.44 & 0.77 & 1.33 & 1.47 & 6.82 \\
7EO & 4.05 & 0.10 & 1.16 & 0.99 & 0.55 & 0.45 & 0.70 & 4.01 \\
9EO & 4.01 & 0.26 & 0.34 & 0.52 & 0.45 & 0.19 & 0.36 & 2.66 \\
11EO & 3.97 & 0.44 & 0.19 & 0.13 & 0.06 & 0.09 & 0.14 & 0.79 \\
14EO & 3.41 & 0.49 & 0.18 & 0.08 & 0.10 & 0.07 & 0.11 & 0.60 \\
20EO & 3.44 & 0.29 & 0.13 & 0.10 & 0.05 & 0.07 & 0.12 & 0.58 \\
25EO & 3.17 & 0.28 & 0.13 & 0.09 & 0.06 & 0.06 & 0.10 & 0.50 \\
$\tau / \mathrm{S}$ & 0.016 & 0.074 & 0.34 & 1.59 & 7.39 & 34.3 & 159.2 & $\infty$
\end{tabular}

Satisfactory fits were found with $\mathrm{i}=7$ and with the longest and shortest relaxation times $\tau_{1}$ and $\tau_{\text {i }}$ equal to the inverse of the highest and lowest radial frequency used. The remaining $\tau_{\mathrm{p}}$ values were logarithmically divided between $\tau_{1}$ and $\tau_{i}$. This leaves eight parameters $\left(G_{0}\right.$ to $\left.G_{7}\right)$ to be fitted. ${ }^{15}$ Although in principle an exact solution should be obtainable, since all free parameters arelinear, this kind of fit is known to be "ill posed", ${ }^{16}$ with many possible solutions. We therefore used a nonlinear curvefit procedurein which we minimized thelogarithmic least-squares sum of $G^{\prime}$ and $G^{\prime \prime}$. By minimization of the squared sum of the logarithms, no weighing of the data is required to obtain a good fit. Only positive parameter values were allowed. The drawn lines in Figure 3 were calculated with the obtained parameters. The result of thecurvefit is given in Table2. Notethat $G$ at theshortest relaxation time $\left(G_{1}\right)$ is almost equal for all samples and does not depend on the mor phol ogy of the lamellar phase.

The observed step change in microstructure is clearly shown in the measured dynamic responses. In Figure 4 the change in $\mathrm{G}_{0}$ and the change in the limiting strain for linear viscoelastic behavior is given as function of $\mathrm{EO}$ chain length. Note that an identical step change is seen in $\mathrm{G}_{4}$ to $\mathrm{G}_{7}$ in Table 2, indicating that the change is especially noticeable at long times (low frequencies).

The explanation of the magnitude of $\mathrm{G}^{\prime}$ at high frequencies is not yet clear. ${ }^{17,18}$ The influence of structure, prestrain, and timescale has to betaken intoaccount, but this has not (yet) been figured out in a complete theory. For thetime being, threestill relevant estimations can be given. If $\mathrm{G}^{\prime}$ is determined by the relaxation of multilamellar spherical droplets ${ }^{17}$

$$
\mathrm{G}^{\prime} \propto \frac{\sigma_{\text {eff }}}{\mathrm{R}}
$$

where $\sigma_{\text {eff }}$ is the effective surface tension and $\mathrm{R}$ is the radius of the spheres. Here $\sigma_{\text {eff }} \sim 10^{-5} \mathrm{~N} / \mathrm{m}^{17}$ and $\mathrm{R} \sim 500$ $\mathrm{nm}$; thus $\mathrm{G}^{\prime} \sim 20 \mathrm{~Pa}$, close to the value found for $2 \mathrm{EO}$ to $9 \mathrm{EO}$ and $11 \mathrm{EO}$ to $25 \mathrm{EO}$ after shear.

If $G^{\prime}$ is determined by interactions between thelamellae, then, in affine deformation, $G^{\prime} \sim B$, where $B$ is the compressibility modulus. Since B is on order of 1000 $\mathrm{Pa},{ }^{17,18}$ this is much larger than the measured value of $\mathrm{G}^{\prime}$. The last explanation assumes a prestrain $\gamma_{0}$ for asymmetriconions, leading to $G^{\prime} \sim B \gamma_{0}{ }^{2}{ }^{18}$ Since $\gamma_{0}$ is unknown, this can always be brought into agreement with experimental results.

3.2.2. Nonlinear Experiments. Typical examples of viscosity as function of shear rate are given in Figure 5. Thecurvefor sample4EO is representativefor samples

(15) Mackley, M. R.; Marshall, R. T.J .; Smeulders, J . B. A. F .; Zhao, F. D. Chem. Eng. Sci. 1994, 49, 2551.

(16) Honerkamp, J . Rheol. Acta 1989, 28, 363.

(17) van der Linden, E.: Dröge, J . H. M. Physica A 1993, 193, 439.

(18) Panizza, P.; Roux, D.; Vuillaume, V.; Lu, C.-Y. D.; Cates, M. E. Langmuir 1996, 12, 248.

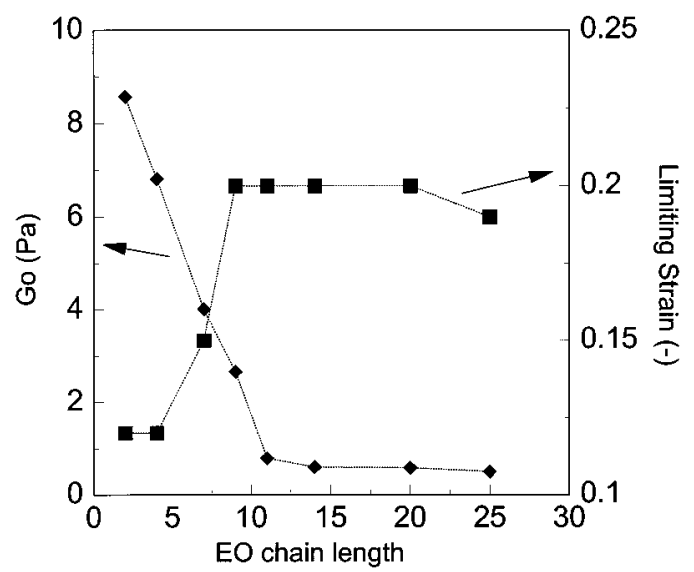

Figure 4. $\mathrm{G}_{\mathrm{o}}$ obtained by fit $(\diamond)$ and the limiting strain for linear viscoel astic behavior ( $\boldsymbol{\square})$ as function of $\mathrm{EO}$ chain length. Lines are drawn to guide the eye.

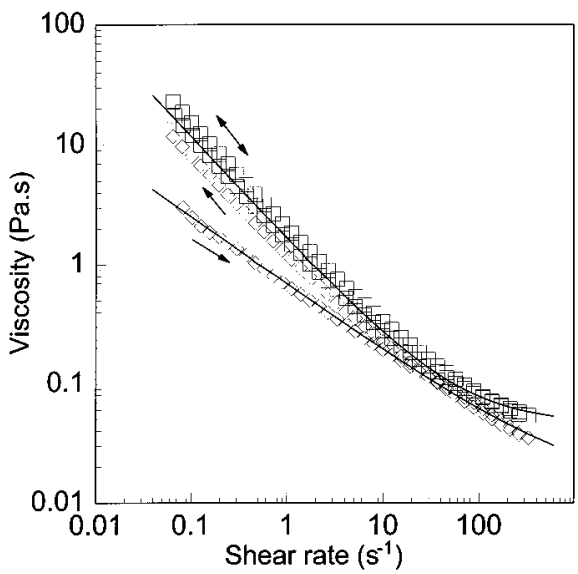

Figure 5. Flow curves for sample 4EO $(O)$ and 14EO $(\diamond)$, arrows indicate the direction of the change of the shear rate.

up to $9 \mathrm{E} \mathrm{O}$. The samples $11 \mathrm{EO}$ to $25 \mathrm{EO}$ behave as $14 \mathrm{E} \mathrm{O}$ does. The samples from $2 \mathrm{EO}$ to $9 \mathrm{EO}$ show no difference between theup-curveand the down-curve, indicating that, in the range of shear rates examined, no detectable, persisting changes in mi crostructure occur. The samples with $11 E O$ up to $25 \mathrm{EO}$ show a noticeable shear/time history. Thefirst up-curveis bel ow thesubsequent downand up-curves, indicating a detectable change in microstructure due to the shear history.

All samples areshear-thinning, with a tendency towards a Newtonian plateau at high shear rates $\left(\eta_{\infty}\right)$. The data was fitted to the Siskoequation with a nonlinear curve-fit procedure. The Sisko equation is a simplified version of the general Cross $^{19}$ flow model:

$$
\eta=\eta_{\infty}+\frac{\eta_{0}-\eta_{\infty}}{1+\alpha \dot{\gamma}^{(1-n)}}
$$

Since we could not detect a first Newtonian plateau $\left(\eta_{0}\right)$, theequation can besimplified. For $\alpha \dot{\gamma}^{(1-n)} \gg 1$, eq 5 reduces to

$$
\eta=\eta_{\infty}+\mathrm{K}_{\dot{\gamma}^{(\mathrm{n}-1)}}
$$

The parameters in this equation are the viscosity at the high shear rate Newtonian plateau, $\eta_{\infty}(/ \mathrm{Pa} \cdot \mathrm{s})$, the consistency, $\mathrm{K}\left(/ \mathrm{Pa} \cdot \mathrm{S}^{\mathrm{n}}\right)$ and the power law index $\mathrm{n}(/-)$. The drawn lines in Figure 5 are calculated from the fitted parameters. Theresults of thecurvefit aregiven in Tables

(19) Cross, M. M. J . Colloid Sci. 1965, 20, 417. 
Table 3. Parameters from Curve Fit to Sisko Model, First Flow Curve, Starting at Low Shear Rate (Standard Deviation Given in Parentheses)

\begin{tabular}{cccc}
\hline sample & $\eta_{\infty} / \mathrm{Pa} \cdot \mathrm{s}$ & $\mathrm{K} / \mathrm{Pa} \cdot \mathrm{s}^{\mathrm{n}}$ & power law index, $\mathrm{n}$ \\
\hline 2EO & $0.061(0.001)$ & $2.18(0.014)$ & $0.096(0.004)$ \\
4EO & $0.046(0.001)$ & $1.65(0.011)$ & $0.145(0.004)$ \\
7EO & $0.029(0.001$ & $1.87(0.016)$ & $0.144(0.005)$ \\
9EO & $0.030(0.001)$ & $1.57(0.014)$ & $0.165(0.005)$ \\
11EO & $0.017(0.002)$ & $0.74(0.008)$ & $0.403(0.006)$ \\
14EO & $0.013(0.002)$ & $0.68(0.007)$ & $0.431(0.006)$ \\
20EO & $0.018(0.001)$ & $0.60(0.005)$ & $0.367(0.005)$ \\
25EO & $0.016(0.001)$ & $0.53(0.005)$ & $0.396(0.005)$
\end{tabular}

Table 4. Parameters from Curve Fit to Sisko Model, Fourth Flow Curve, Starting at High Shear Rate (Standard Deviation Given in Parentheses)

\begin{tabular}{cccc}
\hline sample & $\eta_{\infty} / \mathrm{Pa} \cdot \mathrm{s}$ & $\mathrm{K} / \mathrm{Pa} \cdot \mathrm{s}^{\mathrm{n}}$ & power law index, $\mathrm{n}$ \\
\hline 2EO & $0.057(0.001)$ & $2.31(0.013)$ & $0.123(0.004)$ \\
4EO & $0.040(0.001)$ & $2.07(0.014)$ & $0.147(0.004)$ \\
7EO & $0.028(0.001$ & $1.95(0.016)$ & $0.140(0.005)$ \\
9EO & $0.029(0.001)$ & $1.65(0.015)$ & $0.151(0.005)$ \\
11EO & $0.031(0.001)$ & $1.38(0.014)$ & $0.184(0.006)$ \\
14EO & $0.029(0.001)$ & $1.19(0.013)$ & $0.202(0.007)$ \\
20EO & $0.024(0.001)$ & $0.89(0.010)$ & $0.221(0.007)$ \\
25EO & $0.023(0.001)$ & $0.85(0.010)$ & $0.223(0.007)$
\end{tabular}

3 (up-curves) and 4 (down-curves). Both $\eta_{\infty}$ and $\mathrm{K}$ decrease with increasing EO chain length upto 11EO. For 14EO to 25EO these values appear to reach a plateau. For samples 2EO to 9EO nolarge differences arefound in the curve-fit parameters between the up- and down-curves. For samples 11EO to 25E O, both $\eta_{\infty}$ and K arelarger after shear. The power law index $\mathrm{n}$ is smaller and reduces due to shear from n-values of about 0.4 to 0.2 . The power law index as function of $\mathrm{EO}$ chain length for an unsheard and a sheared sample is given in Figure 6.

For multilamellar vesicles, $\mathrm{H}$ offmann et al. ${ }^{5}$ found that the yield stress $\left(\sigma_{\mathrm{y}}\right)$ is about 10 times smaller than $\mathrm{G}^{\prime}$. We found a comparable ratio. The significance of this comparison between a linear and nonlinear quantity comes from the following. The modulus at high frequency, multiplied with thelimiting strain for viscoelastic behavior $\left(\gamma_{\text {lim }}\right)$, gives an estimate for the stress at which plastic flow begins. Wehavecompared this quantity (from a pl ot of $\mathrm{G}^{\prime}$ at $1 \mathrm{~Hz}$ as function of strain) to the dynamic yield stress $\left(\sigma_{\mathrm{y}}\right)$ deduced from the flow curves at low rates of shear. The estimate of the dynamic yield stress is only significant when the slope of the flow curve in a double logarithmic plot approaches -1 . This is the case for samples 2EO to 9E O. The result of this comparison is given in Figure 7. This figure shows that both quantities are in fair agreement with each other for a given sample, as one would expect if the plastic flow to flow transition takes place via one type of structural change.

Microscopic observation after shear shows that the samples with continuous lamellar phase undergo a transition todroplets. F or samples $2 \mathrm{E} O$ to $9 \mathrm{E} O$, nochange in microstructure could be detected. The microscopic appearance after shear for all samples is well represented by the top left image in Figure 2.

3.2.3. Linear after Nonlinear Experiments. We have further investigated this change in microstructure through shear by shearing at a predefined rate for $300 \mathrm{~s}$ followed by a frequency sweep. During preshearing the viscosity of the sample was recorded every $15 \mathrm{~s}$. Even at the lowest shear rate, the viscosity reading was constant after at most $45 \mathrm{~s}$. This indicates that the change in morphol ogy of thelamellar phaseduetoshear is completed within this time span and occurs quite rapidly. G' at 0.01 $\mathrm{Hz}$, after the cessation of a given shear rate, is pl otted in Figure 8 for two samples. Since we anticipate that the

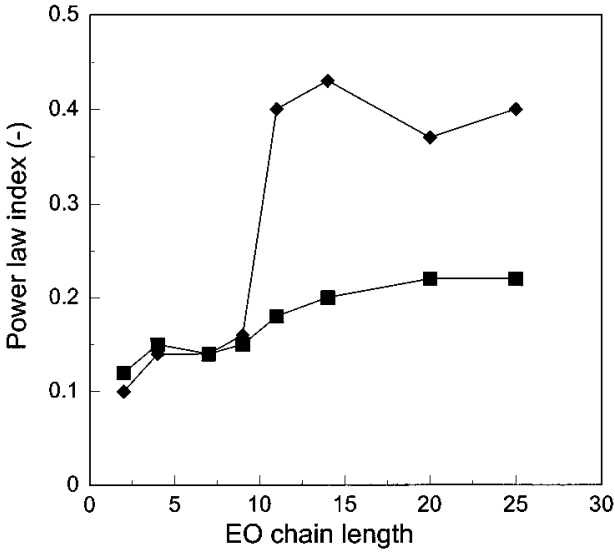

Figure 6. Power law index as function of EO chain length: $(\bullet)$ cal culated from up-curves; ( $\boldsymbol{\square}$ ) cal culated from down-curves. Lines are drawn to guide the eye.

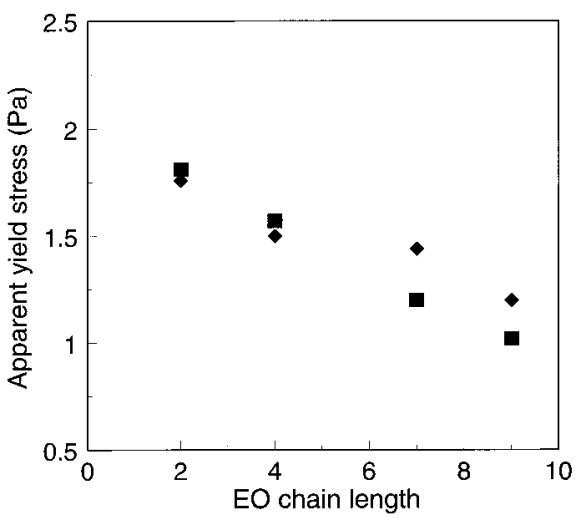

Figure 7. Apparent yield stress as function of $\mathrm{E} O$ chain length, estimated from $\mathrm{G}^{\prime} \gamma_{\lim }(\square)$ and from low shear rate viscosity measurements $(\diamond)$.

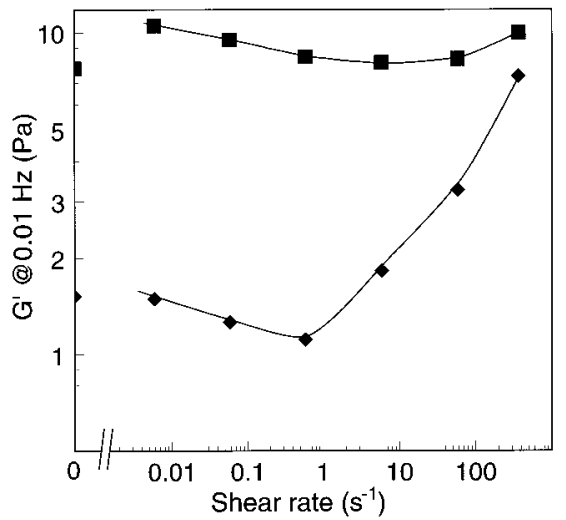

Figure 8. $\mathrm{G}^{\prime}$ at $0.01 \mathrm{~Hz}$ after shear for sample 4EO ( $\square$ ) and $14 \mathrm{EO}(\diamond)$ as function of the preshear rate. A preshear time of $300 \mathrm{~s}$ was used at each rate.

changein $\mathrm{G}^{\prime}$ reflects changes in microstructure, wededuce from this graph that there is no large change in the microstructure of the 4EO sample (representative for samples 2EO to 9EO). The microstructure of 14EO (representative for samples $11 \mathrm{EO}$ to 25EO) starts to changeal ready at shear rates as low as $1 \mathrm{~s}^{-1}$. Theobserved initial decrease in $\mathrm{G}^{\prime}$ for $14 \mathrm{EO}$ is probably caused by incomplete structure relaxation before the start of the experiment or by alignment of the lamellar phase due to the imposed shear. ${ }^{20}$ Note that $\mathrm{G}^{\prime}$ after shear is similar for all sampl es because of the comparable microstructure of the samples after shear. 132

(20) Läuger, J .; Linemann, R.; Richtering, W. Rheol . Acta 1995, 34, 


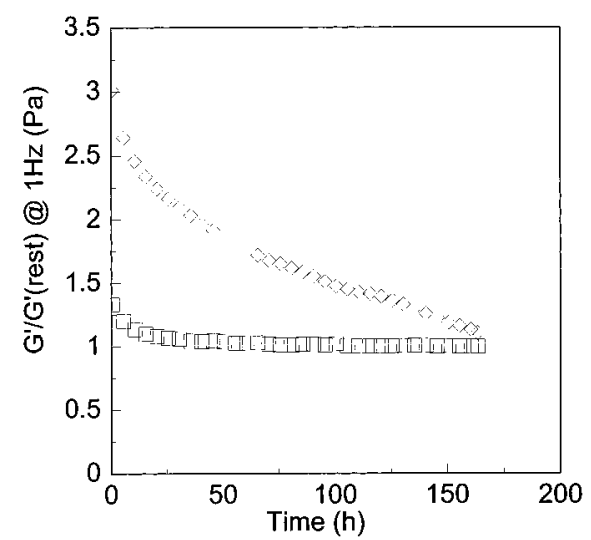

Figure 9. Recovery of $\mathrm{G}^{\prime}$ at $1 \mathrm{~Hz}$ after shear (300 s at $367 \mathrm{~s}^{-1}$ ) for sample $4 \mathrm{EO}(\square)$ and 14EO $(\diamond)$ as function of time.

We also examined the relaxation of the structure after shear (for $300 \mathrm{~s}$ at $367 \mathrm{~s}^{-1}$ ) by measuring $\mathrm{G}^{\prime}$ at $1 \mathrm{~Hz}$ for an extended period of time. Theresults aregiven in Figure 9 , as $\mathrm{G}^{\prime} / \mathrm{G}^{\prime}$ (at rest). For $4 \mathrm{E} O$ weseesomeinitial relaxation possibly caused by the rearrangement of the droplet structure. The sample with 14EO relaxes back to the initial level in about 1 week.

The picture that emerges is that when the lamellar phase is primarily present as lamellar droplets, the power law index is between 0.1 and 0.2 . When the structure changes to platelike continuous lamellar, the power law index increases to 0.35 or 0.4 . After shear, the power law index of the latter samples becomes approximately 0.2 due to a reversible change in microstructure from continuous lamellar to lamellar droplets.

\section{Conclusions}

Lamellar phases, prepared from anionic and nonionic surfactants, can be quite insensitive to small changes in the average EO chain length of the nonionic surfactant used. When to the base composition, containing $6 \%$ anionic, 3\% 7 EO nonionic, and 10\% NTA, was added $1 \%$ of $E O$ nonionic with an average $E O$ chain length of 2,4 , 7, or 9, equal lamellar phase morphology and comparable rheol ogical properties were found. Thelamellar phase is present as a dispersi on of multilamellar droplets (onions). When to the base mixture was added $1 \%$ nonionic with an average $E O$ chain length of $11,14,20$, or 25 , mainly continuous lamellar phases with again similar morphology and rheological properties are found.

A drastic and abrupt change in lamellar phase morphology and in rheological properties occurred on going from a $9 E O$ nonionicto an $11 E O$ noni oni c surfactant. The change in the limiting strain for linear viscoelastic behavior occurs already at lower EO chain lengths. We have no explanation for this. The change in chemical composition by exchanging $1 \%$ of the $9 \mathrm{E} O$ nonionic surfactant for $1 \%$ of the $11 \mathrm{EO}$ nonionic surfactant is very small. It merely means that the distribution of EO chain lengths is shifted marginally. Despitethis small change, the morphology changes from dispersed lamellar to primarily continuous lamellar. And as a consequence of that also the linear and nonlinear rheological properties changed considerably. Continuous lamellar phase can be converted to dropl ets by shear at rates above $1 \mathrm{~s}^{-1}$. The droplets will relax back in about 1 week. Shape fluctuations of lamellar droplets, which were seen using confocal laser microscopy wereassociated with a relaxation process detected in the measured dynamic moduli.

Acknowledgment. Wewould liketothank Mr. André van der Salm for experimental assistance and Dr. Erik van der Linden for many stimulating discussions on the subject of lamellar phases. We also want to thank Mr. Marcel Paques, Mr. Michiel Leunis, and Mr. Han Blonk for the electron microscopy work and the confocal laser microscopy work. They also contributed considerably to the interpretation of the images obtained with these techniques.

\section{LA9702603}

